\section{Explanations of cold fusion}

SIR-I suggest that electrolysis of a heavywater solution of deuterated lithium hydroxide with a palladium cathode leads to the formation of a higher deuteride of palladium, probably $\mathrm{PdD}_{2}$ with the fluorite structure, the subsequent decomposition of which leads to the increased evolution of heat or to the explosion of the deuteride into palladium powder and hydrogen gas.

In a paper in Physical Review in $1938 \mathrm{I}$ pointed out that in the transition metals 0.72 orbital per atom of the orbitals in the outer shell remains apparently unused. Later, I identified the 0.72 orbital per atom as the metallic orbital, required for the unsynchronized resonance of covalent bonds that confers metallic properties on a substance. The principal evidence for the metallic orbital is that the curve of the saturation paramagnetic moments of the iron-group metals has a foot at the composition $\mathrm{Ni}_{44} \mathrm{Cu}_{56}$. I also pointed out that the need for the metallic orbital for stability explains the fact that palladium is saturated with hydrogen at the composition $\mathrm{PdH}_{0.6}$. Introduction of additional hydrogen atoms leads to instability because of the loss of resonance energy when the metallic orbital is not available.

I judge that under the conditions of the electrolysis experiments of M. Fleischmann and S. Pons (J. electroanalyt. Chem. 261, 301-308; 1989) deuterons beyond this limit are forced into the palladium cathode, ultimately forming the unstable higher deuteride. Later, after the beginning of electrolysis, this unstable deuteride may begin to decompose either slowly, resulting in an increased liberation of heat, or explosively, as Fleischmann and Pons observed when a 1-cm cube of the deuterated palladium disappeared.

It can be predicted that, because of the difference in amplitude of the zero-point vibrations of the nuclei with different masses, palladium dihydride would be less stable than palladium dideuteride, and palladium ditritide would be more stable - perhaps stable enough to permit examination by $\mathrm{X}$-ray diffraction.

LINUS PAULING

Linus Pauling Institute of Science and Medicine,

440 Page Mill Road,

Palo Alto, California 94306, USA

SiR-Hydrogen in metals may tend to segregate to cracks, grain boundaries or interfaces and to dislocations. This is a well-known phenomenon and it explains several important observations such as hydrogen-induced fracture in ferritic steels. We suggest that prior accumulation of hydrogen in defects in palladium electrodes may prevent the build-up of deuterium, and that this could explain why cold fusion is hard to replicate.
The equilibrium segregation of hydrogen can be characterized as a function of the binding energy and of the local stress for the special case of a crack. The quantity of segregated hydrogen, $\left(C_{\mathrm{H}}\right)_{\mathrm{j}}$ is given by

$$
\left(C_{\mathrm{H}}\right)_{\mathrm{i}}=\frac{C_{\mathrm{m}} \exp \left(\left(\Delta H_{\mathrm{B}}\right)_{\mathrm{ef}} / k T\right)}{1-C_{\mathrm{m}}+C_{\mathrm{m}} \exp \left(\left(\Delta H_{\mathrm{B}}\right)_{\mathrm{ef}} / k T\right)}
$$

For a surface, grain boundary, interface or dislocation, the effective binding energy of hydrogen is $\left(\Delta H_{\mathrm{B}}\right)_{\mathrm{ef}}=\left(\Delta H_{\mathrm{B}}\right)_{\mathrm{j}}$, whereas for a crack-tip it is $\left(\Delta H_{\mathrm{B}}\right)_{\mathrm{ef}}=$ $\left(\Delta H_{\mathrm{B}}\right)_{\mathrm{j}}+\sigma_{1} V_{\mathrm{H}}$. In these equations, the binding energy is $\Delta H_{\mathrm{B}}$ and the local stress is $\sigma_{1}$. The subscript $\mathrm{j}$ indicates the segregation site of the hydrogen (for example, $\mathrm{s}$ for crack surfaces and b for boundaries). $C_{\mathrm{m}}$ is the bulk hydrogen content, $V_{\mathrm{H}}$ is the molar volume of hydrogen, $k$ is Boltzmann's constant and $T$ the absolute temperature. $\left(\Delta H_{\mathrm{B}}\right)_{\mathrm{j}}$ can be described by the standard chemical potentials of hydrogen and the metallic matrix in which it segregates, at the segregation site and remote from it. Thus the magnitude of $\left(\Delta H_{\mathrm{B}}\right)$, depends on the type of hydrogen segregation site ${ }^{1}$.

We suggest that when in a cold fusion experiment a palladium electrode is immersed in a heavy water-based electrolyte, the deuterium diffuses into the palladium and segregates to dislocations and other defects according to the above equation. When the concentration in these local regions has reached a high enough level, the reaction commences in the segregated region. We are unable to say just what the reaction is, but it may be a form of nuclear fusion or some chemical reaction. The period of electrolysis that precedes the reaction is attributed to the build-up of deuterium to the critical concentration in the regions around the dislocations or other defects in the palladium.

If, during the period before the experiment commences, hydrogen has diffused into the palladium and segregated to the dislocations, there will be fewer lattice interstitial sites into which the deuterium can migrate when electrolysis in heavy water commences, and the deuterium concentration around the defects may never attain a high enough level for the 'fusion' reaction to start.

If this hypothesis is correct, it accounts for the failure of some laboratories to produce the 'fusion' reaction described by Fleischmann and Pons ${ }^{2}$. The solution is to purify the palladium before electrolysis so as to remove hydrogen and any other interstitial atoms that, by segregating to the crystal defects, prevent the critical

1. Kameda. J. Res Mechanica 26, 215-249 (1989).

2. Fleischmann, M. J.\& Pons. S. J. electroanalyt. Chem. 261, $301-308$ (1989) concentration of deuterium from being attained in the experiments.

UK Atomic Energy Authority, 11 Charles II St, London SW1Y 4QP, UK

JAMES BOCKRIS

Texas A\&M University,

College Station, Texas 77843, USA

\section{Stereo-image}

SiR-Vance Tucker (Nature 337, 605; 1989) notes that few readers keep stereoscopes handy and that only a quarter of those he surveyed could view stereo-pairs without one.

The main function of a stereoscope is to counteract the normal correlation between the focus and convergence muscles in the eye. Its lenses bring the focus to the printed page while the eye muscles are focused and converged at infinity, with the left eye viewing the lefthand image, and the right eye the right.

On the other hand, I wear glasses for near-sightedness to bring objects at infinity into focus, as my unaided eyes maximal focus distance is much closer. Thus I and other near-sighted readers can create a stereoscope by merely removing our glasses.

3-28-8-412 Yaguchi, Ota-ku, Tokyo 146, Japan

\section{Trilobite attacks}

SIR-Babcock and Robison' report that sublethal predation scars on Palaeozoic trilobites occur about three times as frequently on the right side of the trilobite body as on the left. They conclude that the predators of the period apparently preferred to attack the trilobite's right side, and that this provides evidence of behavioural asymmetry and brain laterality in the fossil record. I would like to suggest an alternative explanation, related to the trilobite's defensive response to attack.

Many trilobite fossils are found curled up ${ }^{2}$. Curling probably enabled trilobites to protect their more vulnerable ventral surface, in a manner perhaps analogous to that of modern-day terrestrial isopods such as garden slaters (woodlice or sow bugs). Picture the following. Before the trilobite completes the curl, the middle lateral margins are raised off the substrate as the head (prosoma) or tail (pygidium) is curled beneath the body. Since the head shield provides the greatest protective surface, curling would probably have started at the tail. A balanced, bilaterally symmetrical, curling motion would simultaneously lift both lateral margins off the substrate as the body is arched so that sublethal predation scars would be expected to be equally frequent on both sides. This would also be the case if the trilobite toppled over onto one side as curling 